\title{
An Efficient Fabric Defect Prediction Based on Modular Neural Network Classifier with Alternative Hard C-Means Clustering
}

\author{
S. Rathinavel ${ }^{1 *}$, T. Kannaianl ${ }^{2}$ \\ ${ }^{1}$ Assistant Professor, Dept of Electronics and Instrumentation, Bharathiar University, Coimbatore. \\ ${ }^{2}$ Associate Professor, Department of Electronics, PSG College of Arts \& Science, Coimbatore. \\ *Corresponding authorE-mail:rathinavel@buc.edu.in
}

\begin{abstract}
In India, textile industry has been mainly focused because it increased the economy day by day. But, it has some problem in the field of quality control. At present, it is mainly solved visually through skilled workers. Though, due to the human errors and eye fatigue, the system reliability has been restricted. So, in this research has been focused automatic fabric defect detection scheme. Here, Modular Neural Network (MNN) is proposed for fabric defect detection and classification with low cost and high accurate rate via using image processing schemes in the woven fabrics. At first, the images are collected from the machine and then preprocessed by using Enhanced Directional Switching Median Filter (EDWF) to reduce the impulse and stationary noise. To attain high accurate prediction, the preprocessed image has been segmented by using Alternative Hard C-Means (AHCM) cluster. After clustering, the images are converted to binary image. Then, the first order features has been extracted from the image. The extracted features are given as input to MNN, which classifies the fabric defects. In MNN, the weight factors are calculated by using back propagation algorithm and generate the output. The simulation results show that the proposed MNN attained high accuracy rate of $96.7 \%$ when compared to existing Artificial Neural Network (ANN) than Support Vector Machine with Genetic Algorithm (SVM-GA) classification algorithms.
\end{abstract}

Keywords: Fabric defect, textile industry, modular neural network, C-Means cluster, median filter, segmentation, classification.

\section{Introduction}

A major anxiety for fabric industries is identifying defects in fabric and is most important for quality products. Particularly, in fabric field, detect the defects is most important task because of the usually used material in daily life. In garment industry [1], the $85 \%$ of the defects occurred by the fabric defects only. It's reduced the turnover of the industry by $45-65 \%$ [2]. Therefore, to detect and predict these defects from reoccurring is very important. Presently, the defects are identified by visual human inspection, but it's not efficient due to the human errors and eye fatigue and it attain success rate of $60-75 \%$. So, the automatic defect detection system has been used to reduce the labor cost and improve the quality in textile industry. Generally, more than 70 types of defects $[3,4]$ are there in textile industry. The automatic fabric defect detection system has been detected most possible defects and achieved more reliable system with high quality control. In this work, the automatic system with image processing scheme based fabric defect detection has been introduced.

In textile industry, the defect has been identified by human only. So, this process has been taken high time and labor cost. Initially, the production machine produced the adequate amount of fabric and then removed from the machine and batched into larger rolls and then sent to the visual human inspection. Here, the production has been taken long time as well as the inspection also taken same time. For that reason, the income of the industry has been reduced. To solve this problem, the automatic system has been mainly focused. This system has been prevented production of the defects and improved the production quality or changed the process parameters to predict the defect automatically. All the above process are done by using image processing schemes and identifying the faults in fabric as well as the faults are classified by using some classification algorithms.

A variety of defects are occurred in fabrics like weaving defects, texture pattern violation and yarn misplacements etc. in this automatic detection system, the image processing schemes like preprocessing, segmentation and feature extractions has been used. The preprocessing is nothing but noise reduction scheme for improving the image quality. The segmentation is used splitting the affected region from normal regions for improving the defect prediction and classification accuracy. The feature analysis has been used for reducing the processing time. Tuceryan [5] has been defined five major types of features for texture analysis like geometrical, statistical, structural, spectral features and model based features. Chan [6] has been identified the defects as well as classified by using some extracted parameters or features. For real time defect detection in industrial applications, the specialized hardware and faster decision making time also required. So, this work proposed high efficient classification to reduce the response time.

In this fabric field, many research works has been introduced, but still required a robust real time system for color fabrics. In real time, to reduce the response time efficient algorithms are needed. Also, the automatic system has some challenges. To solve this problems some researchers has been introduced and these techniques are predicted in [7]. The objective of this work has been to reduce the response time via using the efficient MNN classification scheme. To improve the image quality and reduce the processing time, the image has been preprocessed. Then, the defect regions are segmented from the image. After that, the 
features are extracted for MNN analysis. Finally, the defects are classified efficiently. The simulation results demonstrate that the proposed MNN achieved better performance compared than existing schemes. The below sections are followed as: part 2 reviewed the existing fabric defect detection schemes, part 3 explained the proposed fabric defect detection and classification, part 4 shows the simulation results of proposed scheme and part 5 concludes the paper.

\section{Literature Survey}

In this part, the existing fabric defect detection has been discussed and it is a popular research topic $[8,9]$ for many years. Navneet Kaur [10] presented a Gabor filter method for textile fault detection problem. It was chosen as a appropriate delegate of this class of schemes. Here, the optimized 2D Gabor filter has been used to provide support of their suitability for this fault detection. Xie Xianghua [11] focused visual inspection system by using image processing schemes, particularly, the texture analysis schemes. Increasing demand of texture analysis for color images has been discussed and given some analysis. Abdellah et al., [12] proposed Support Vector Machine (SVM) with Genetic Algorithm (GA) based classification scheme for defect detection in textile industry. In SVM, the kernel parameters are optimized by using GA. The simulation results show that that acquired $94.84 \%$ of detection rate. Eldeeb et al., [13] presented a computer vision based defect detection system by using Artificial Neural Networks (ANN). The input has been processed and classified defects like weft defect and wrap defect. Mohammed Sathik [14] presented a Bit-Plane slicing to extract the RGB colors of the Original image and it compared with Bit level of gray scale X-Ray image. High degree calculation of periodicity for textile fabrics, it was not suitable. Latif-Amet et al. [15] presented a defect detection method based on the wavelet theory and co-occurrence matrix and classification done by using Mahalanobis distance in textile images. In fabric defect detection, the Gabor filter schemes have been used. It has two filtering process like filter banks as $[16,17]$ and optimal filters $[18,19]$. This filtering with a filter bank has been given high excessive data for process. A model based schemes has been used for fabric defect detection and reduce the time consumption. Markov random field model [20] based texture analysis has been focused to improve detection accuracy. Campbell et al. [21] a model based clustering method scheme has been used to identify linear pattern production defects. Huang and Chen [22] proposed A Back Propagation Neural Network (BPNN) with fuzzy logic based scheme for fabric defect classification with 8 different types. Kumar [23] proposed a Feed-Forward Neural Network (FFNN) based segmentation scheme for local textile defects and given better performance. Jing et al. [24] presented a two-layer neural network based machine visual system for detect surface defect. It has been used patch statistics from raw image data.

\section{Proposed Methodology}

The proposed system step by step process has been depicted Fig 1 . The main aid of this proposed scheme is detects the textile defects in real world. The proposed system has two parts like detect the faults and classified the defects. In first part, the input image is processed and identified the defect. In second part, the identified defect has been classified like dye spot, holes, ladder, pin marks, flat, burst selvedge in textile industry using MNN. Initially, the fabric vibration free images are obtained in inspection system. The obtained image has been preprocessed by using filtering scheme and then normalized. After that, the preprocessed image has been segmented the defect region by using AHCM clustering algorithm and then the image converted into binary images. Then, the grey level concurrence matrix features has been extracted and given as an input to the MNN. In MNN, 78 defected and not defected images are trained and 6 types of fabric have been identified with rate of $96.7 \%$ when compared to existing schemes.

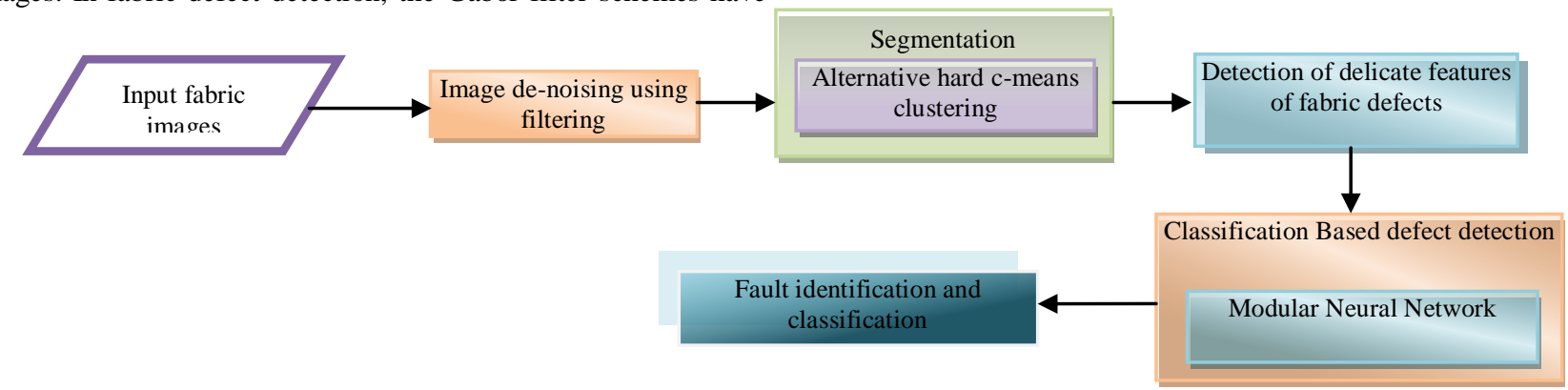

Fig. 1: Overall process of proposed fabric defect detection system

\section{Fabric Material with Light Intensity Optimization}

In vision control system, the inadequate and excess illumines are considered as a major problem and it solved by optimized the light intensity. Initially, the cotton fabric light intensity has been optimized with constant dense and same thickness and it maintain over the process. After that, the penetrated light intensities are captured as images by camera for each weave mark. Then, the same process has been followed for synthetic fabric with size of $256 \times 256$. Finally, the same procedure followed in silk fabric. It is a soft material, so the optimization procedure has been presented more carefully. In this process, first the image has been acquired and then various schemes of process are applied. But, if the acquired image is not satisfied then the proposed tasks may not be attainable even using image enhancement. The textile fabric image has been attained by using CCD camera. That acquired input color fabric images are processed into MATLAB using image processing schemes.
Preprocessed to Noise Removal: one form of image has been converted into another form means, it can be affected by some noise and it can be reduced the image quality. So, some filtering schemes have been introduced. Here, the image is affected by stationary noise and impulsive noise. Here, the adaptive filter has been used to remove stationary noise. Then, Enhanced Directional Switching Median Filter (EDSMF) is applied [25] to reduce impulse noise. The steps are given below

1. Enhanced directional detector has been used detect the impulse noise from input image $\mathrm{X}$.

2. Then, the binary image has been created, which detect the noisy and noise free pixels.

3. EDSMF has been applied to remove noise.

The proposed EDSMF method enhances the traditional Vector Median Filter (VMF) through combining it with adaptive directional detector for impulse noise removal in noisy fabric image. For the efficient access, the image data normalized by 0 and 1.

Image Segmentation Using AHCM Clustering 
Generally, image segmentation scheme has been used for predicting the boundaries like lines, curves, etc. In this process, a label assigned for every pixel in an image, which pixels has same label share their visual characteristics. Here, the clustering based AHCM segmentation has been used. It clusters the defect free region into one cluster and defected region into another cluster. It is a good clustering method and it will cluster data set $X=$ $\left\{x_{1}, \ldots, x_{n}\right\}$ into $\mathrm{c}$ well partitions with $2 \leq c \leq n-1$. While, it has no priori information about unlabelled data set $X$, so it is need an objective function for clustering process. Naturally, each cluster shall be as compact as possible. Thus, AHCM clustering objective function is proposed as

$J_{A H C M}=\sum_{i=}^{c} \sum_{j \in I_{i}}\left\{-\exp \left(-\beta\left\|x_{j}-z_{i}\right\|^{2}\right)\right\}$

Where $\beta$ is a constant and it can be represented as

$\beta=\left(\frac{\sum_{j=1}^{n}\left\|x_{j}-\bar{x}\right\|^{2}}{n}\right)^{-1}$ with $\bar{x}=\frac{\sum_{j=x_{j}}^{n}}{n}$

And $\quad j \in I_{i} \quad$ if $\quad 1-\exp \left(-\beta\left\|x_{j}-z_{i}\right\|^{2}\right)=\min _{k}\{1-$ $\left.\exp \left(-\beta\left\|x_{j}-z_{k}\right\|^{2}\right)\right\} \forall k=1, \ldots, c$. It is defined as constant in order to fix the effect of parameter by using data points. It has been set to be the inverse of sample covariance, and the minimization condition of AHCM objective function is defined as

$z_{i}=\frac{\sum_{j \in I_{i}}\left(\exp \left(-\beta\left\|x_{j}-z_{i}\right\|^{2}\right) x_{j}\right)}{\sum_{j \in I_{i}} \exp \left(-\beta\left\|x_{j}-z_{i}\right\|^{2}\right)}$

The exact AHCM clustering algorithm along with this necessary condition has been discussed given below

Step 1: some initial values $z_{1}, \ldots, z_{c}$ has been chosen

Step $2 \mathrm{n}$ data points are classified by assigning them to the class of the smallest distance $d(x, y)=-\exp \left(-\beta\|x-y\|^{2}\right)$

Step 3: new centers $z_{i}$ has been obtained by using the fixed-point iteration. Condition check if no new $z_{i}$ is found, then stop the process. Otherwise go to step 2.

If the necessary condition can't be solved directly, then iterative method has been used to attain step 2. Thus the segmentation region is localized, which is to find out the smallest rectangle that can completely contain the defect area.

\section{Feature Extraction}

To improve the classification accuracy, the grey level histogram based features has been extracted. It defines the statistical distribution of image grey value. Seven types of features has been extracted such as energy $H_{p}$, grey average $\bar{l}$, entropy $H_{e}$, variance $\sigma^{2}$, maximum difference of grey value $\mathrm{G}$ and the maximum grey value $G_{m}$ using the formula given in [26]. Additionally, the geometric feature has been extracted and it has the information's related to the shape. Here, the first step is defined as the binarization of the defect image. Then, six types of feature parameters has been extracted such as width $\mathrm{W}$, length $\mathrm{L}$, the ratio of width and length $\mathrm{B}$, perimeter $\mathrm{C}$ area $\mathrm{S}$ and dispersion $\mathrm{A}$. width has been represented as the vertical width of the defect area and is computed by the vertical projection. Length has been represented the horizontal length of defect area and is computed by horizontal projection. Generally, the ratio of width and length has been described the degree of weft of the defect. Then, the area has been represented the sum of all values which has grey value 1 in the binary image. The perimeter has been represented the sum of all pixels in the region of the segmentation edge. The measure of the defect shape has been represented as dispersion.

\section{The Fabric Defect Classification and Identification Using MNN}

The fabric defect detection last step is defect identification and classification. This process has been done using extracted features on the fabric defects. In this work, one of the most important statistical methods of MNN has been used for classification.

Modular Neural Network: generally, a computation system, which has two or more subsystems that can be worked, based on the same or various inputs independently are named as the modularity. In MNN, two or more individual neuron modules has been comprised, which can be independently act on the inputs and produced output. In this neural network, the Divide and conquer scheme has been absorbed a number of advantages like flexibility in design and implementation, scalability, complexity reduction, computational efficiency and robustness. While considered a problem with a large number of dimensions solved by these properties and make modeling very efficiently and easily. In MNN, three stages have been considered and are shown in fig 2 . In this process, the features are given to the input to $\mathrm{MNN}$ and it divided in to modules and then classification decision has been predicted. Here, 6 types of defected classes are defined. Initially, the problems are split into smaller problems and then each one sent to one of the modules. Here, the task decomposition process has been introduced and it has been take a look at the artificial data and is depicted in fig 3 . Here, the input space has been split into two regions like two classed of data such as crosses and circles is defined in upper right. Then, three classes of data represented like above two classes with triangle. These two classes with samples have belonging to the two different regions. The defect classifier has been given to all regions and transformed this particular problem into two simpler ones. In this training phase, each and every modules has been trained until it solves the particular sub problems. Then, the decision phase has been introduced to produce a final network output. Generally, it can be done by using a gating network, module vote has been makes or hierarchical integration has been used. In this work, in the task decomposition phase, a

gating network has been used and it considers additional modules, which trained to identify the region of input space wherever each of the modules have their region of expertise. After completion of learning process, a new pattern is given to the network, the individual module calculates the classes, and particular output is selecting by gate network. The training process of the network defines the optimization of network detection performance. It has been identified via minimizing the performance function. The Mean Squared Error (MSE) has been defined as the performance function. Finally, the classification results has been predicted among $[-1,1]$ and predicts whether the block region contain defect region or not.

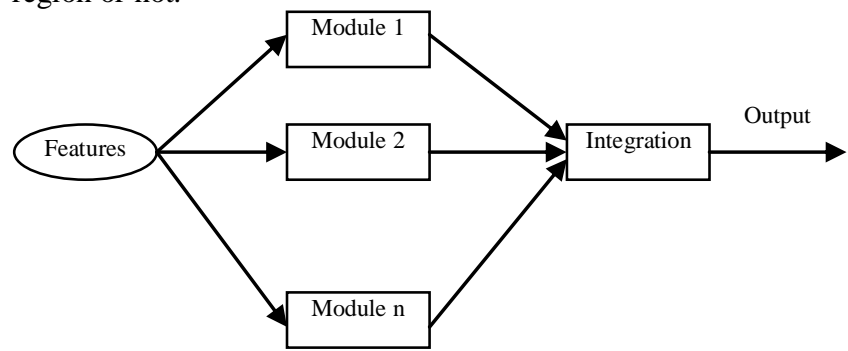

Fig. 2: Overall process of a modular neural network 


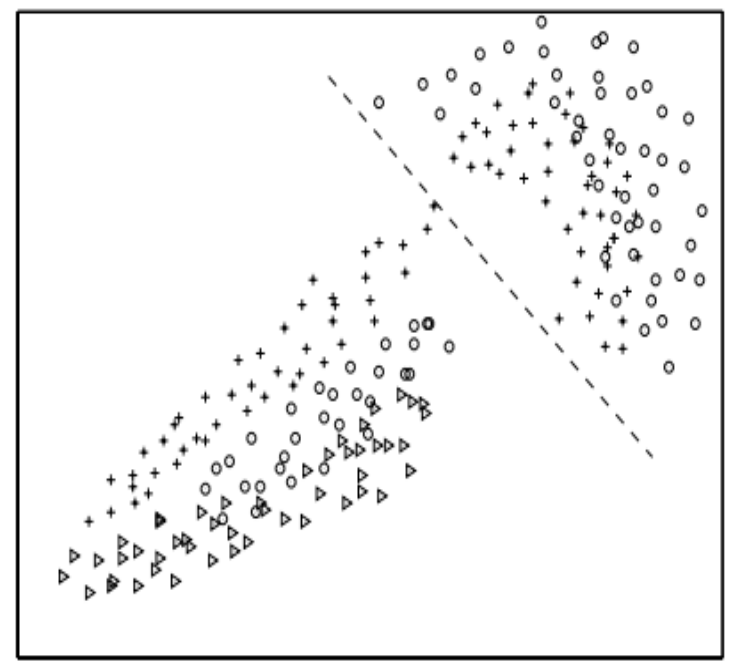

Fig. 3: The partition of the input space for a 3 class problem

\section{Experimental Results and Discussion}

This section presents the experimental results for fabric defect detection. In this section, the real time collected dataset has been used to analyze performance results compared with existing ANN [13] and SVM-GA [12] schemes using MATLAB.

\section{Dataset}

The fabric defect images have been captured from visual system by using CCD cameras in real time. It contains 100 images and 75 are used for training and 25 used for testing process. Here, once the MNN is trained in the learning phase (i.e offline step) it will be inserted in the defect detection process and then the global
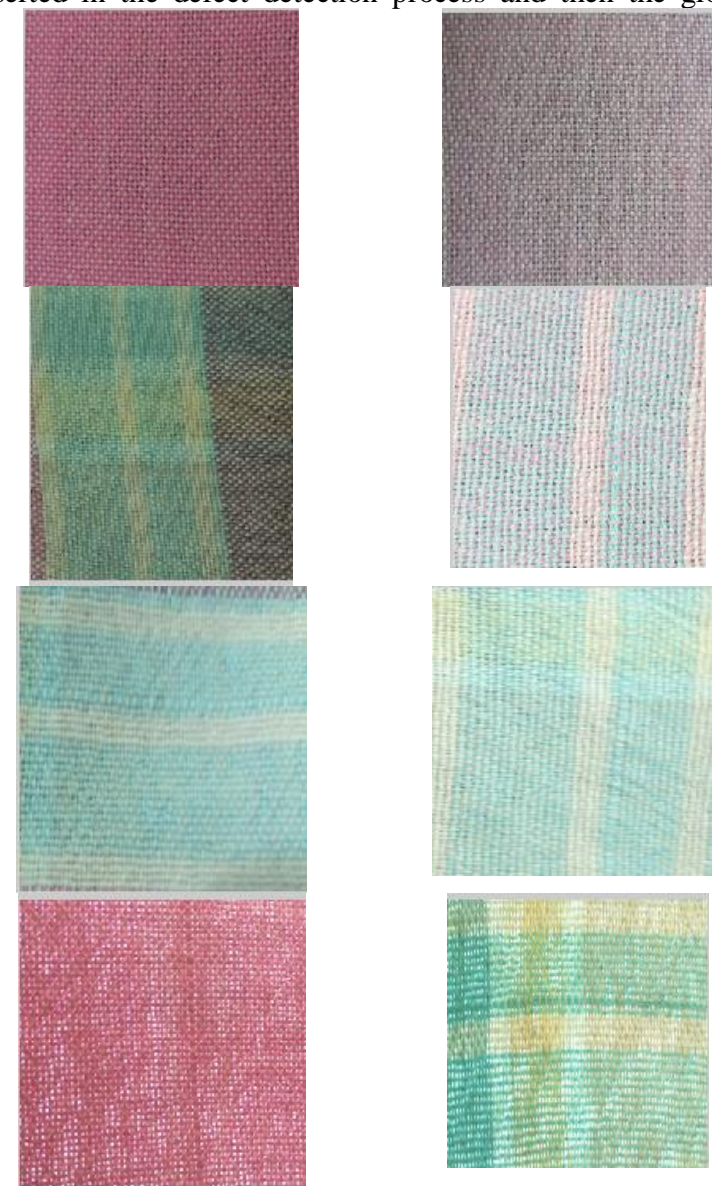

needed time is considerably reduced. The training process has been carried out with error BP by using set of fabric images with defect 52 and without defect 23 images. Initially, the weight values of $\mathrm{NN}$ have been set among 0 and 0.1 and learning coefficient is set as 0.6 .

\section{Performance Discovery}

After the MNN is trained with 75 images, the remaining 25 images have been tested. The performance of trained and tested images has been measured separately in terms of classification accuracy, processing time, convergence speed, MSE and PeakSignal-to-Noise Ratio (PSNR). The accuracy has been measured among the total number of correctly classified test samples and total number of test samples and is defined as

$\operatorname{Accuracy}(\%)=\frac{T P+T N}{T P+F N+T N+F P} \times 100$

Where TN-true negative (i.e wrongly identified correct sample), TP-true positive (i.e correctly identified sample), FP-false positive (i.e the defect sample correctly identified) and $\mathrm{FN}$-false negative (i.e defect sample identified wrongly). In the training process, all the MNN classifiers achieve an average accuracy of $96.7 \%$ in the whole dataset. This indicates that the classifiers are well trained and can be applied for fabric defect detection. Though, in the testing process, these classifiers are validated beside the test data and the average accuracy is about $96.7 \%$ for original features. For image the time needed for defect detection through scanning the whole image is about 0.8 seconds. Taking into account the production rate, the algorithm can be used in a real time application.
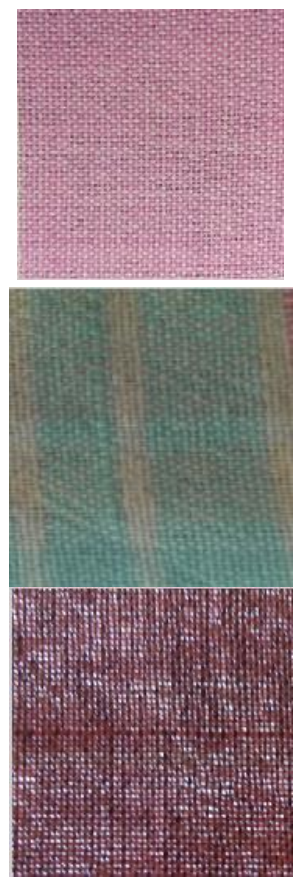

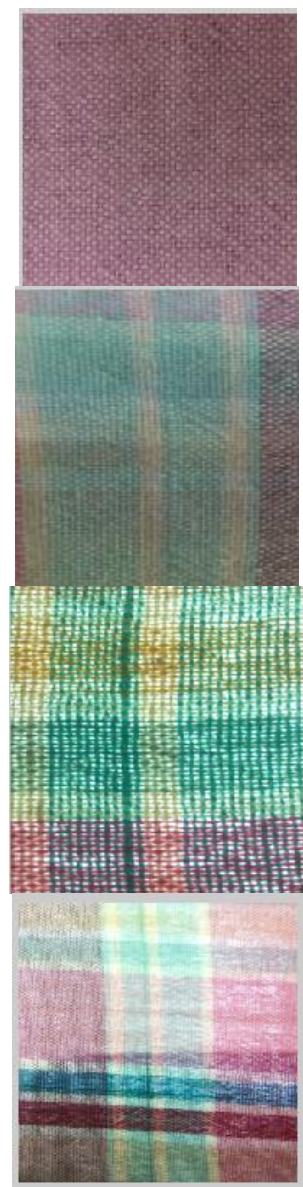



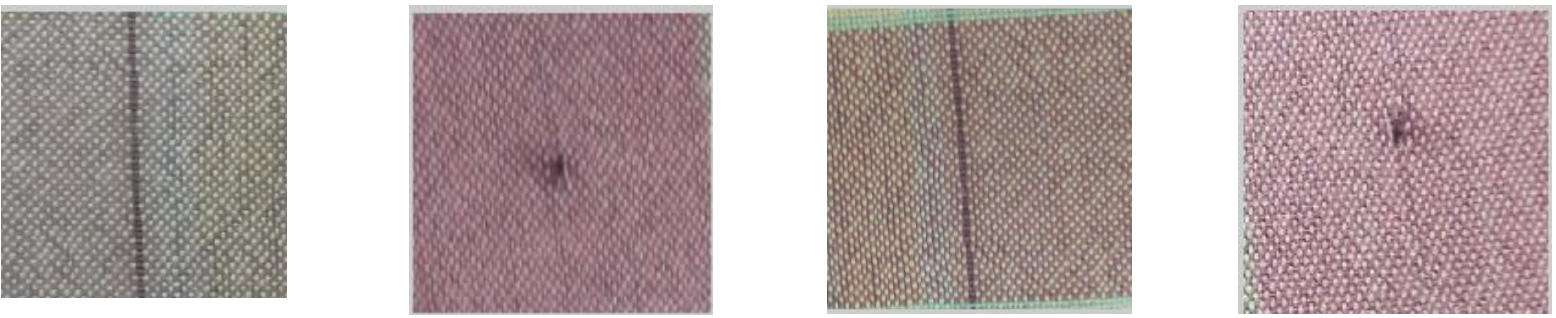

Fig. 4: Fabric dataset samples

\section{Discussion and Comparison with Existing Classification Methods}

In this experiment, the image is attained after scanning the input image using a window of size pixels. After applying the segmentation, the features extractions are generated through dividing the fabric image into overlapping blocks of size pixels. Finally, the classification result is obtained from a modular network trained. Fig 4 shows examples of the fabric sample dataset.

Usually, these kinds of fabric defects are varied in shape, size and orientation. In that situation, the proposed algorithm has been managed to predict most of the defects in accurate manner. Based on the accurate prediction result, the proposed MNN concluded that it can be effectively improved the classification performance in textile industry. Finally, the evaluation of proposed MNN has been attained good potential result for improving fabric defect detection compared than existing ANN and SVM-GA showed in table 1 . The average accuracy result is $96.7 \%$ due to the efficient feature extraction and classifier.

Table 1: Classification Accuracy for All Classification Algorithms

\begin{tabular}{|c|c|c|c|c|c|c|c|c|}
\hline Number of images & $\mathrm{S} V \mathrm{~V} \mathrm{M}-\mathrm{G}$ & & A & $\mathrm{N}$ & $\mathrm{N}$ & $\mathrm{M}$ & $\mathrm{N}$ & $\mathrm{N}$ \\
\hline 5 & 8 & 4 & 8 & & 5 & 8 & & 9 \\
\hline 1 & 8 & 6 & 8 & & 8 & 9 & 0 & 5 \\
\hline 1 & 8 & 9 & 9 & & 1 & 9 & & 2 \\
\hline 2 & 9 & 0 & 9 & & 2 & 9 & & 4 \\
\hline 2 & 9 & 1 & 9 & & 3 & 9 & & 5 \\
\hline 3 & 9 & 2 & 9 & 4 & 5 & 9 & 6 & 7 \\
\hline
\end{tabular}

\section{Accuracy Performance Comparison}

Fig 5 shows that the accuracy performance comparison results for proposed MNN and existing ANN than SVM-GA. It illustrate that the proposed MNN attained high accuracy rate compared than existing classifiers due to the efficient segmentation process and feature extraction. When the number of images will increase means the accuracy also increased.

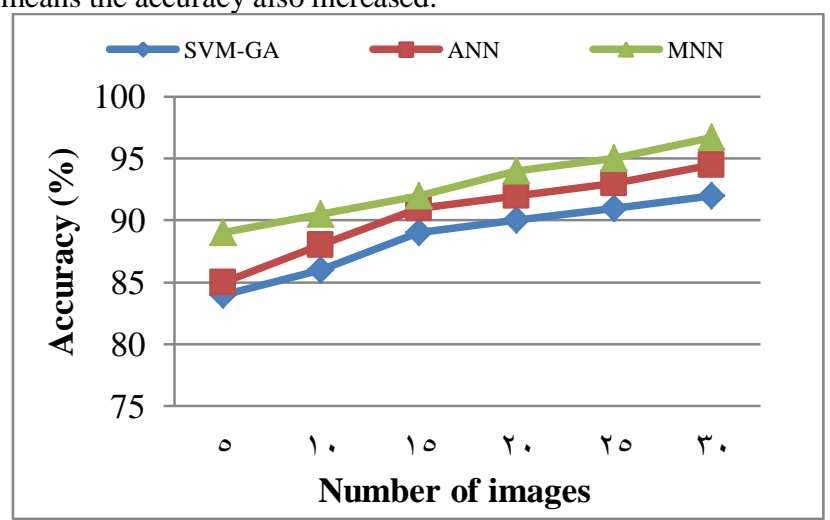

Fig. 5: Accuracy performance comparison vs. number of images

\section{Convergence Speed Performance Comparison}

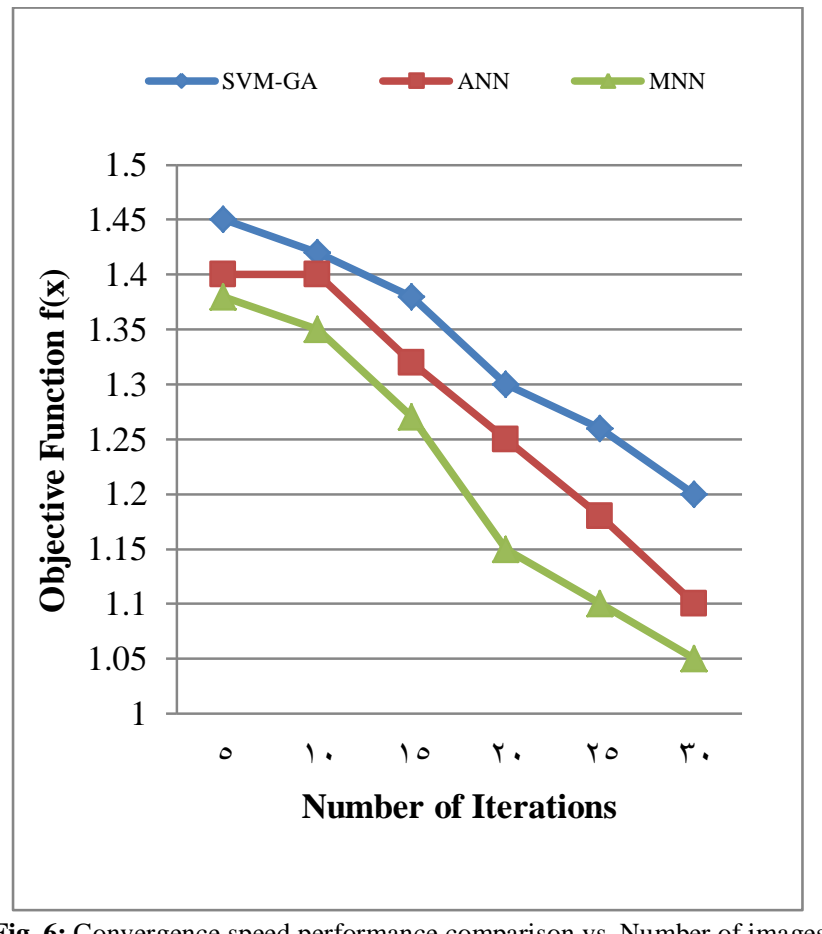

Fig. 6: Convergence speed performance comparison vs. Number of images

The convergence speed performance comparison among proposed MNN and existing ANN, SVM-GA is showed in Fig 6. The narrow span of this graphical representation shows the proposed MNN attained high convergence speed based on the objective function compared than existing scheme. The numerical evaluation is showed in table 2.[27]

Table 2: Convergence Speed Numerical Evaluation for All Classifiers

\begin{tabular}{|c|c|c|c|c|c|c|c|c|c|}
\hline Number of images & & M - & & $\mathbf{A}$ & $\mathbf{N}$ & $\mathbf{N}$ & $\mathbf{M}$ & $\mathbf{N}$ & $\mathbf{N}$ \\
\hline 5 & 1 & 4 & 5 & 1 & . & 4 & 1 & 3 & 8 \\
\hline 1 & 1 & 4 & 2 & 1 & . & 4 & 1 & 3 & 5 \\
\hline 1 & 1 & 3 & 8 & 1 & 3 & 2 & 1 & 2 & 7 \\
\hline 2 & 1 & & 3 & 1 & 2 & 5 & 1 & 1 & 5 \\
\hline 2 & 1 & 2 & 6 & 1 & 1 & 8 & 1 & & 1 \\
\hline 3 & 1 & & 2 & 1 & & 1 & 1 & 0 & \\
\hline
\end{tabular}




\section{PSNR performance comparison}

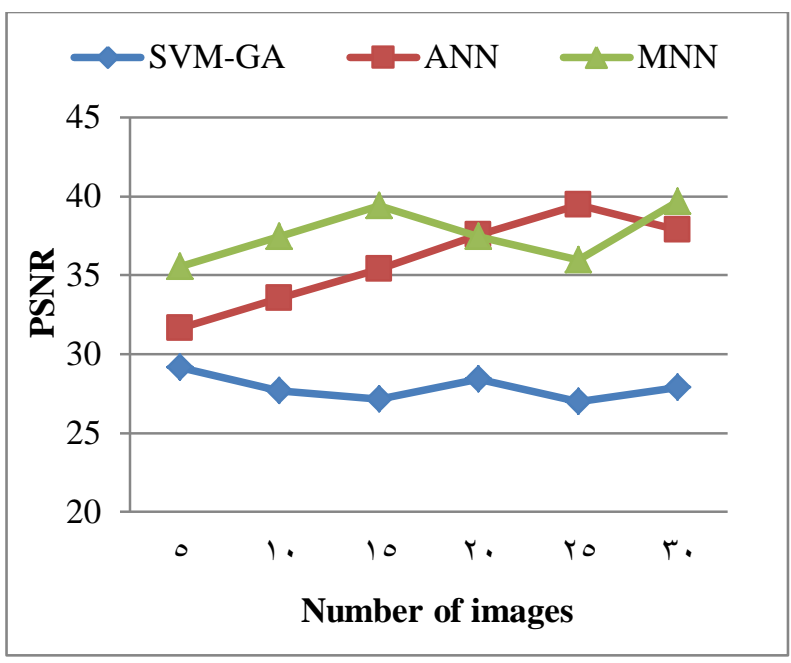

Fig. 7: Comparison of PSNR in various classification algorithms

Fig 7 shows that the PSNR performance comparison evaluation results among the proposed MNN and existing ANN and SVMGA. It illustrate the proposed MNN attained high PSNR rate compared than existing classifiers due to the less MSE and less processing time. The numerical evaluation is showed in table 3 .

Table 3: PSNR Numerical Evaluation for All Classifiers

\begin{tabular}{|c|c|c|c|c|c|c|c|}
\hline Number of images & S $\quad V \quad M \quad G \quad G$ & & & $\mathbf{N} \quad \mathbf{N}$ & & $\mathbf{M}$ & $\begin{array}{ll}\mathbf{N} & \mathbf{N} \\
\end{array}$ \\
\hline 5 & $\begin{array}{llllll}2 & 9 & . & 1 & 6 & 5 \\
\end{array}$ & & 31 & 6546 & & 5 & 56689 \\
\hline 0 & 27.6589 & & 33 & 5465 & & 37 & 4699 \\
\hline 5 & 27.1256 & & 35 & $\begin{array}{llll}4 & 1 & 2 & 3 \\
\end{array}$ & & 39 & 4125 \\
\hline 0 & 28.4065 & & 37 & 5648 & & 37 & 4626 \\
\hline
\end{tabular}

\begin{tabular}{|ll|llllll|llllll|llllll|}
\hline 2 & 5 & 2 & 6 & 9 & 8 & 6 & 4 & 3 & 9 & 4 & 4 & 5 & 6 & 3 & 5 & .9 & 5 & 4 & 4 \\
\hline 3 & 0 & 2 & 7 &. & 8 & 7 & 3 & 7 &. & 8 & 7 & 3 & 9 &. & 6 & 7 \\
\hline
\end{tabular}

\section{MSE Performance Comparison}

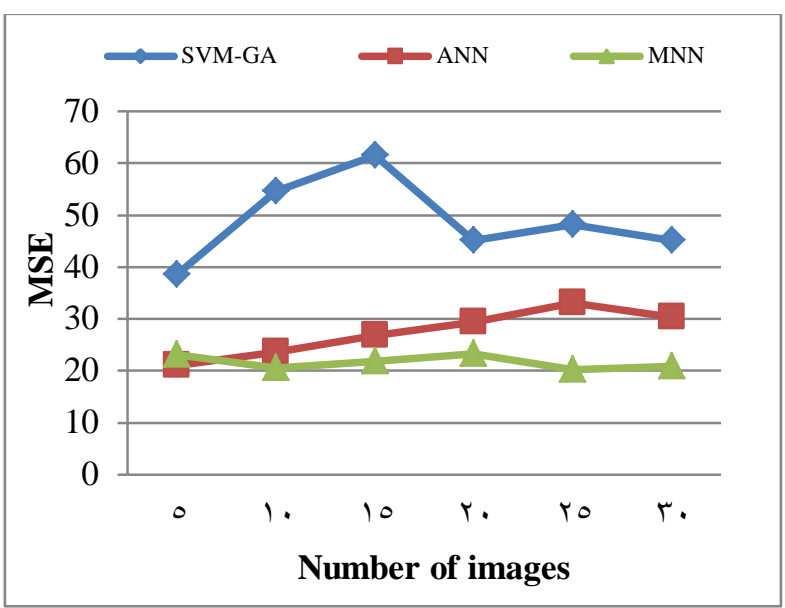

Fig. 8: MSE performance comparison vs. Number of images

Fig 8 shows that the MSE performance comparison results between proposed MNN and existing ANN and SVM-GA. It illustrate that the proposed method has less value of MSE and it indicating the high-quality reconstructed image due to the efficient segmentation. The numerical evaluation results are showed in table 4.

Table 4: MSE numerical evaluation for all classifiers

\begin{tabular}{|c|c|c|c|c|c|c|c|c|c|c|c|c|c|c|c|c|c|c|c|c|c|c|c|c|c|c|c|c|c|}
\hline 1 & $\mathrm{ul} \mathrm{m}$ & $b \quad e$ & $\mathrm{er}$ & $\begin{array}{ll}0 & 1 \\
\end{array}$ & $\mathrm{i}$ & $\mathrm{m}$ & a & g $\quad \mathrm{e}$ & s & $S$ & $\mathrm{~V}$ & M & & - & G & $\mathbf{A}$ & $\mathbf{A}$ & & & $\mathbf{N}$ & & & $\mathbf{N}$ & $\mathbf{M}$ & & $\mathbf{N}$ & & & $\mathbf{N}$ \\
\hline 5 & & & & & & & & & & 3 & 8 & . & 4 & 4 & 6 & 4 & 2 & 1 & . & & 0 & 3 & 8 & 2 & 3 & 1 & 2 & 5 & 4 \\
\hline 1 & & & & & & & & & 0 & 5 & 4 & . & 5 & 1 & 8 & 9 & 2 & 3 & & 5 & 9 & 5 & 5 & 2 & 0 & 5 & 8 & 4 & 1 \\
\hline 1 & & & & & & & & & 5 & 6 & 1 & . & 5 & 7 & 2 & 7 & 2 & 6 & . & 7 & 2 & 8 & 1 & 2 & 1 & 8 & 5 & 4 & 1 \\
\hline 2 & & & & & & & & & 5 & 4 & 8 & . & 1 & 2 & 4 & 2 & 3 & 3 & . & 0 & 6 & 2 & 6 & 2 & 0 & 2 & 5 & 6 & 1 \\
\hline 3 & & & & & & & & & 0 & 4 & 5 & &. & & 1 & 2 & 3 & 0 & & . & & 3 & 2 & 2 & 0 & & & & 7 \\
\hline
\end{tabular}

\section{Processing time comparison}

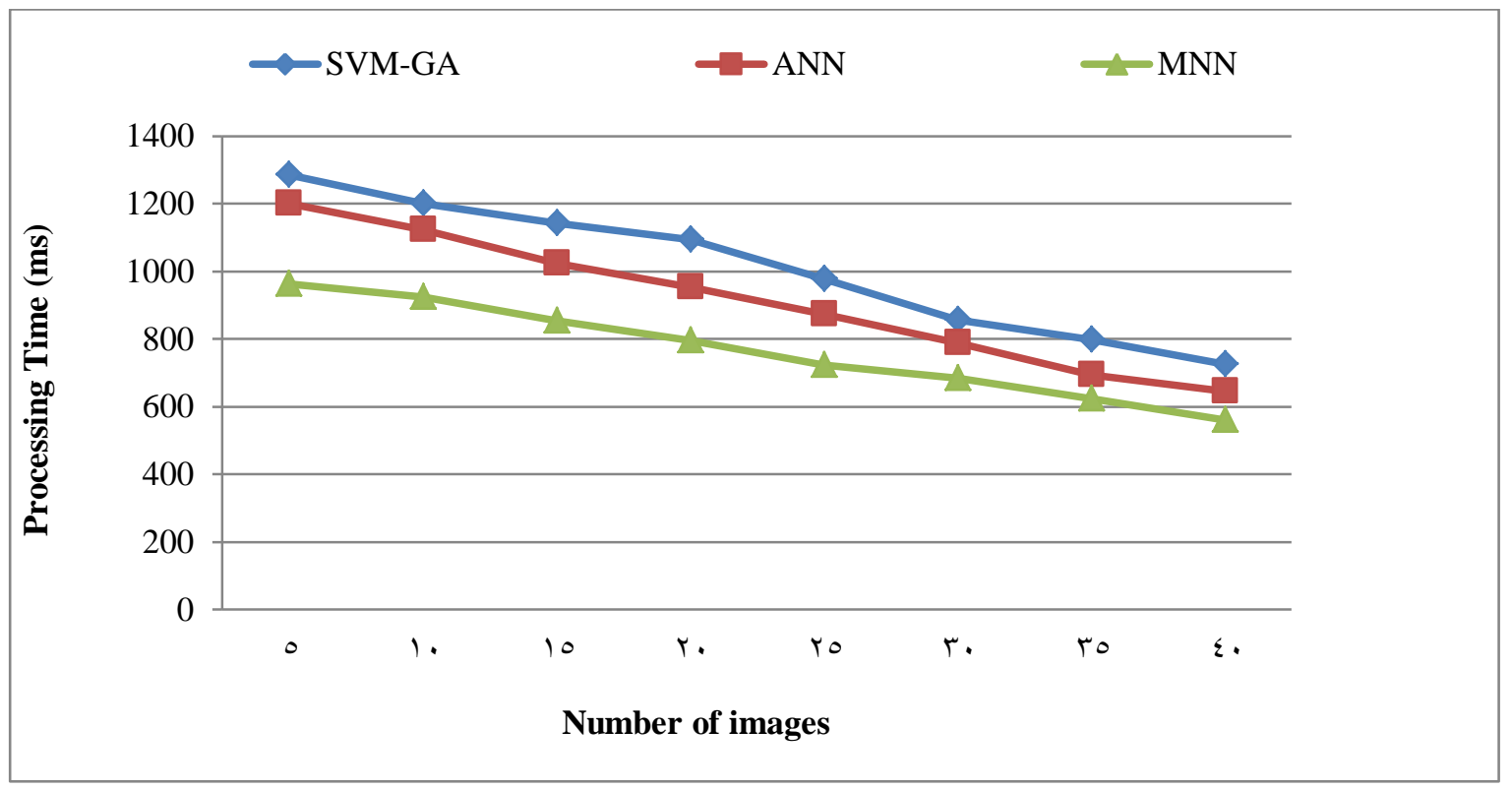

Fig. 9: Processing time vs. number of images 
Fig 9 shows the processing time performance comparison among proposed MNN and existing ANN and SVM-GA. From the figure, it is recognized that the proposed MNN method has taken less time to detect the defect. It is also obvious that, with increasing the images, the processing time becomes higher for all the methods, that is, a better defect detection can be obtained by taking more measurements. The numerical evaluation results are showed in table 5.[28]

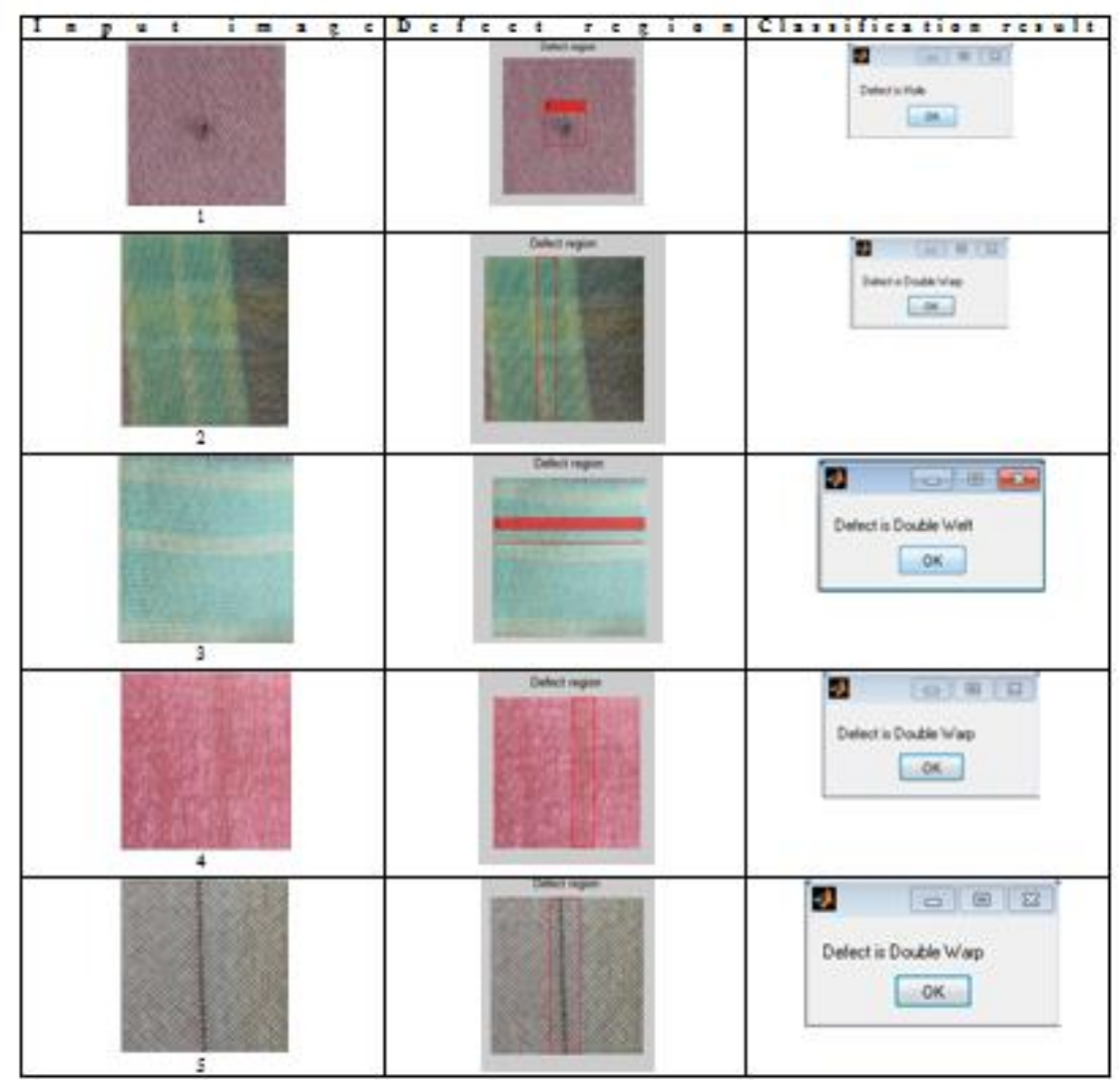

Fig. 10: Defect perdition and classification result for sample dataset images

Table 5: Processing Time Numerical Evaluation for All Classifiers

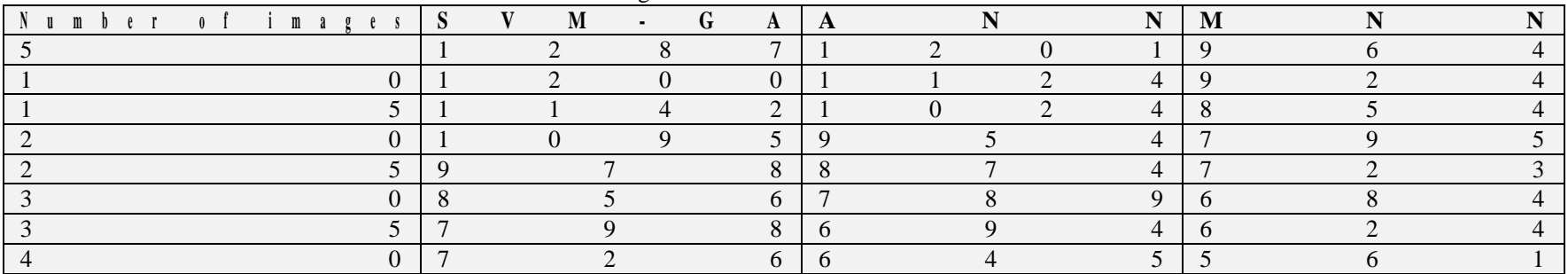

\section{Conclusion}

In this work, Modular Neural Network (MNN) based novel scheme is proposed to resolve the problem of fabric defect detection, which is based on the segmentation and efficient feature extraction. AHCM based image segmentation is done to localize the defects and MNN is proposed to find the defects. The classification performance of this proposed MNN has been widely evaluated via using the real time images of the textile industry. The comparison with other existing algorithm of ANN than SVMGA reported in literature highlights the efficiency of the MNN approach. The accuracy of block condition was $96.7 \%$ and the simulation results demonstrate that the proposed MNN algorithm is a strong technique for fabric defect detection. The grey level and geometric features has been used to effectively classify the defects for a sample of a very large number of high-quality images. The MNNs have a fast convergence speed under general regular conditions and less processing time. But it may have slower convergence rate under relatively weaker conditions. In order to solve this issue, further the adaptive network-based fuzzy inference system based classifier is proposed to detect the defect.

\section{References}

[1] Sengottuvelan P, Wahi A \& Shanmugam A, "Automatic fault analysis of textile fabric using imaging systems", Research Journal of Applied Sciences, Vol.3, (2008), pp.26-31.

[2] Vikrant T \& Gaurav S, "Automatic fabric fault detection using morphological operations on bit plane", International Journal of Engineering Research \& Technology, Vol.2, (2013), pp.856-861.

[3] Hong Kong Productivity Council, Textile Handbook 2000, The Hong Kong Cotton Spinners Association, 2000.

[4] Ngan HYT, Pang GKH \& Yung NHC, "Automated fabric defect detection-a review", Image and Vision Computing, Vol.29, No.7, (2011), pp.442-458.

[5] Tuceryan M \& Jain A, Texture Analysis, The Handbook of Pattern Recognition and Computer Vision, $2^{\text {nd }}$ Edition, World Scientific Publishing Co., (1998), pp.207-248.

[6] Chan C \& Pang G, "Fabric defect detection by Fourier analysis", Conference Record of the IEEE Industry Applications Conference, Thirty-Fourth IAS Annual Meeting, Vol.3, (1999), pp.1743-1750.

[7] Mahajan PM, Kolhe SR \& Patil PM, "A review of automatic fabric defect detection techniques", Advances in Computational Research, Vol.1, No.2, (2009), pp.18-29. 
[8] Chin RT \& Harlow CA, "Automated visual inspection: a survey", IEEE Trans. Pattern Analysis \& Machine Intelligence, Vol.4, No.6, (1982), pp.557-573.

[9] Haralick RM, "Statistical and structural approaches to texture", Proceedings of the IEEE, Vol.67, No.5, (1979), pp.786-804.

[10] Kaur N \& Dalal M, "Application of machine vision techniques in textile (fabric) quality analysis", IOSR Journal of Engineering, Vol.2, No.4, (2012), pp.582-584.

[11] Xie X, "A Review of Recent Advances in Surface Defect Detection using Texture analysis Techniques", Electronic Letters on Computer Vision and Image Analysis, Vol.7, No.3,(2008), pp.1-22.

[12] Abdellah H, Ahmed R \& Slimane O, "Defect detection and identification in textile fabric by SVM method", IOSR Journal of Engineering (IOSRJEN), Vol.4, No.12,(2014), pp.69-77.

[13] Eldeeb H, Mohy, Elbagoury T, Aboseda K, Shady E \& Eldessouki M, "An automated fabric fault detection and classification system based on computer vision and soft computing", ACC Journal, (2013).

[14] Mohammed Sathik M, "Feature Extraction On Colored X-Ray Images By Bitplane Slicing Technique", International Journal of Engineering Science and Technology, Vol.2, No.7, (2010), pp.2820-2824.

[15] Latif-Amet A, Ertuzun A \& Ercil A, "An efficient method for texture defect detection: sub-band domain co-occurrence matrices", Image and Vision Computing, Vol.18, No.6, (2000), pp.543-553.

[16] Kumar A \& Pang GKH, "Fabric defect segmentation using multichannel blob detectors", Optical Engineering, Vol.39, No.12, (2000), pp.3176-3190.

[17] Kumar A \& Pang GKH, "Defect detection in textured materials using Gabor filters", IEEE Transactions on Industry Applications, Vol. 38, No.2, (2002), pp.425-440.

[18] Hamid A, Alireza A \& Esmaeil S, "Defect detection in textiles using morphological analysis of optimal Gabor wavelet", Proceedings of the International Conference on Computer and Automation Engineering, (2009), pp.26-30.

[19] Li M \& Staunton RC, "Optimum Gabor filter design and local binary patterns for texture segmentation", Pattern Recognition Letters, Vol.29, No.5, (2008), pp.664-672.

[20] Ozdemir S \& Ercil A, "Markov random fields and KarhumenLoeve transforms for defect inspection of textile products", Proceedings of the IEEE Conference on Emerging Technologies and Factory Automation, Vol.2, (1996), pp.697-703.

[21] Campbell JG, Fraley C, Murtagh F \& Rafery AE, "Linear flaw detection in woven textiles using model-based clustering", Pattern Recognition Letters, Vol.18, No.14, (1997), pp.1539-1548.

[22] Huang CC \& Chen IC, "Neural-fuzzy classification for fabric defects", Textile Research Journal, Vol.71, No.3, (2001), pp.220 224.

[23] Kumar A, "Neural network based detection of local textile defects", Pattern Recognition, Vol.36, No.7, (2003), pp.1645-165.

[24] Jing F, Li M, Zhang HJ \& Zhang B, "Unsupervised image segmentation using local homogeneity analysis", Proceedings of the IEEE International Symposium on Circuits and Systems, (2003), pp.II456-II459.

[25] Anitha S \& Radha V, "Preprocessing using Enhanced Median Filter for Defect Detection in 2D Fabric Images", International Journal of Engineering and Technology (IJET), Vol.6, No.2, (2014), pp.707717.

[26] Zhong Z, Qi P, Guan M, Xia Y \& Fu Y, "Fabric defect detection system based on digital image processing", Computer Modelling \& New Technologies, Vol.18, No.10,(2014), pp.263-270.

[27] G, Abikhanova, A Ahmetbekova, E Bayat, A Donbaeva, G Burkitbay (2018). International motifs and plots in the Kazakh epics in China (on the materials of the Kazakh epics in China), Opción, Año 33, No. 85. 20-43.

[28] A Mukanbetkaliyev, S Amandykova, Y Zhambayev, Z Duskaziyeva, A Alimbetova (2018). The aspects of legal regulation on staffing of procuratorial authorities of the Russian Federation and the Republic of Kazakhstan Opción, Año 33. 187 216. 